\title{
VISEDU-MAT: um ambiente para o ensino de funções matemáticas nos espaços $\mathbf{R} 2 \mathrm{e} \mathbf{R 3}$
}

\author{
Mauricio Capobianco Lopes ${ }^{1}$, Dalton Solano dos Reis ${ }^{2}$, Viviane Clotilde da Silva ${ }^{1}$, \\ Gabriel Zanluca ${ }^{2}$, Samara Reinert ${ }^{3}$ \\ ${ }^{1}$ Programa de Pós-Graduação em Ensino de Ciências Naturais e Matemática \\ Universidade Regional de Blumenau (FURB) - Rua Antonio da Veiga, 140 - CEP \\ 89.030-903 - Blumenau, SC - Brasil \\ ${ }^{2}$ Departamento de Sistemas e Computação \\ Universidade Regional de Blumenau (FURB) - Blumenau, SC - Brasil \\ ${ }^{3}$ Departamento de Matemática \\ Universidade Regional de Blumenau (FURB) - Blumenau, SC - Brasil \\ mclopes@furb.br, dalton@furb.br, gabriel.zanluca@gmail.com, \\ vcs@furb.br, samarareinert.sa@hotmail.com
}

\begin{abstract}
VISEDU is a framework developed in the Regional University of Blumenau, which provides a software infrastructure for the construction of learning objects (LOs) applied to different fields of knowledge. This article aims to present an LO for teaching mathematical functions in $R 2$ and R3, built from VISEDU. High school students from a State school in Blumenau are testing the web module of VISEDU-MAT. The results showed that students were very motivated with the use of the environment. In conclusion, simple and intuitive tools can assist teachers in teaching and students in understanding about the content of mathematical functions.
\end{abstract}

Resumo. O VISEDU é um framework desenvolvido na Universidade Regional de Blumenau que oferece uma infraestrutura de software para a construção de Objetos de Aprendizagem (OAs) aplicados a diferentes áreas do conhecimento. Este artigo tem por objetivo apresentar um OA para o ensino de funções matemáticas nos espaços $R 2$ e R3, construido a partir do VISEDU. O módulo web do VISEDU-MAT está sendo testado por estudantes do ensino médio de uma escola estadual de Blumenau. Os resultados demonstraram que os estudantes ficaram bastante motivados com o uso do ambiente. Conclui-se que ferramentas simples e intuitivas podem auxiliar os professores no ensino e os estudantes na compreensão sobre o conteúdo de funções matemáticas.

\section{Introdução}

No contexto da criação de softwares de simulação voltados à educação, o Grupo de Pesquisa em Tecnologias Digitais Aplicadas à Educação, vinculado ao Departamento de Sistemas e Computação da Universidade Regional de Blumenau desenvolveu o framework Visualizador de Material Educacional (VISEDU). Em sua versão inicial o VISEDU foi pensado para o ensino de funções matemáticas no espaço R2 [Krauss, 2013]. Machado (2014) e Montibeller (2014) ampliaram as funcionalidades do 
V Congresso Brasileiro de Informática na Educação (CBIE 2016)

Anais dos Workshops do V Congresso Brasileiro de Informática na Educação (CBIE 2016)

VISEDU, com a possibilidade de se trabalhar com câmaras e texturas nas aplicações. Nunes (2014) incorporou elementos de splines e iluminação aos objetos. Atualmente há três Trabalhos de Conclusão de Curso em andamento ampliando as funcionalidades do VISEDU e um projeto de pesquisa de iniciação científica que incorpora a possibilidade de simulação do espaço R3.

O VISEDU tem sido aplicado em diferentes áreas de conhecimento como Matemática, Física e Ciências Naturais. O presente artigo tem por objetivo apresentar um OA para o ensino de funções matemáticas nos espaços R2 e R3, construído a partir do VISEDU, denominado VISEDU-MAT (http://mat.vai.la). Este OA foi aplicado em uma escola de ensino médio da cidade de Blumenau por uma professora de matemática. As próximas seções apresentarão o software e os principais resultados alcançados com a aplicação.

\section{VISEDU-MAT}

O VISEDU-MAT permite a simulação e interação dos estudantes nos espaços R2 e R3. A Figura 1 apresenta a tela do ambiente para o espaço R2.

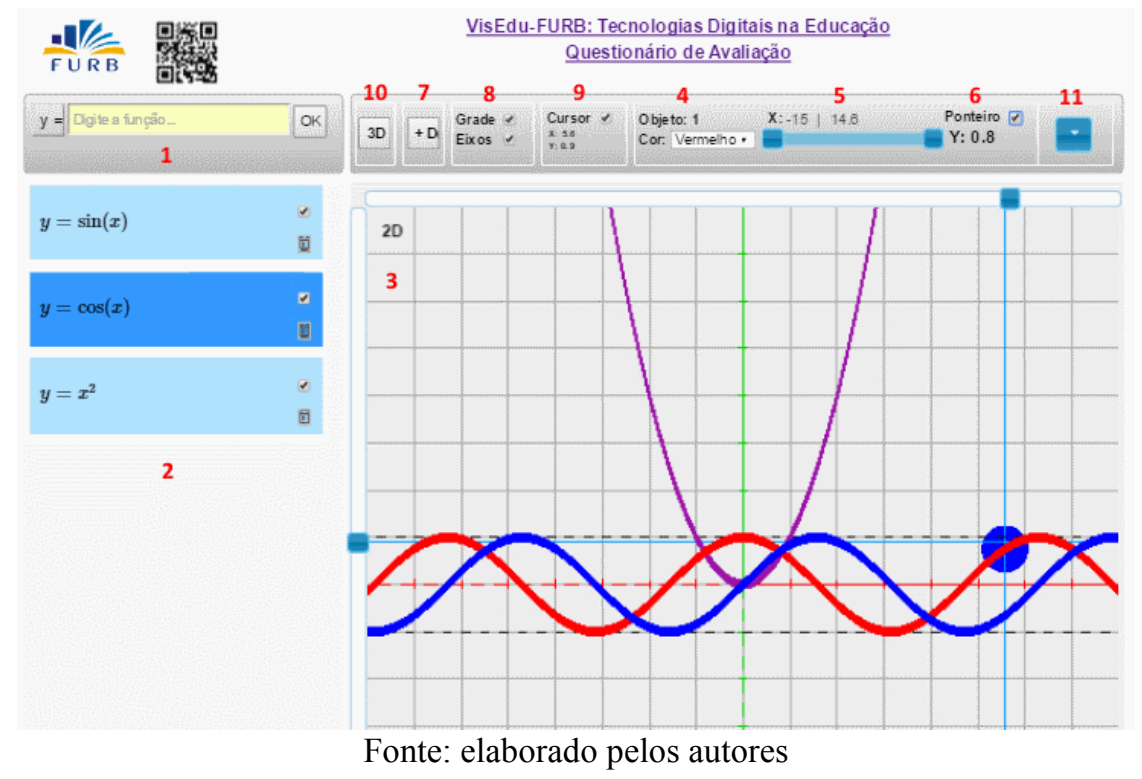

Figura 1. VISEDU-MAT - Espaço R2

O ambiente apresenta as seguintes funcionalidades: (1) digitação da função: espaço para a digitação de uma função matemática. O contradomínio pode ser selecionando clicando no botão $y=$. Para plotar uma nova função deve-se digitá-la no campo de texto e pressionar o botão OK; (2) funções: espaço no qual aparece a representação matemática das funções criadas. Nele é possível desabilitar a exibição da função ou excluí-la; (3) plotagem: espaço de plotagem das funções criadas pelo usuário; (4) cor: permite alterar a cor da função selecionada; (5) X: amplia a área de plotagem da função no eixo do domínio; (6) Ponteiro: indica o valor da coordenada do contradomínio em um determinado ponto da função; (7) +D: melhora a qualidade da plotagem do gráfico; (8) grade e eixos: habilita ou desabilita a visualização da grade e do eixo do plano cartesiano; (9) cursor: indica a coordenada x e y da posição do cursor do mouse no plano cartesiano; (10) 3D: muda o ambiente para a simulação de funções 
V Congresso Brasileiro de Informática na Educação (CBIE 2016)

Anais dos Workshops do V Congresso Brasileiro de Informática na Educação (CBIE 2016)

no espaço R3; (11) menu: apresenta exemplos didáticos para o uso do ambiente e ajuda para o uso dos recursos e funções.

A Figura 2 apresenta o ambiente no espaço R3. Neste espaço as opções são ampliadas para mais um eixo. Neste caso, é possível fazer a rotação dos eixos e a função pode ser selecionada a partir de um clique duplo no gráfico.

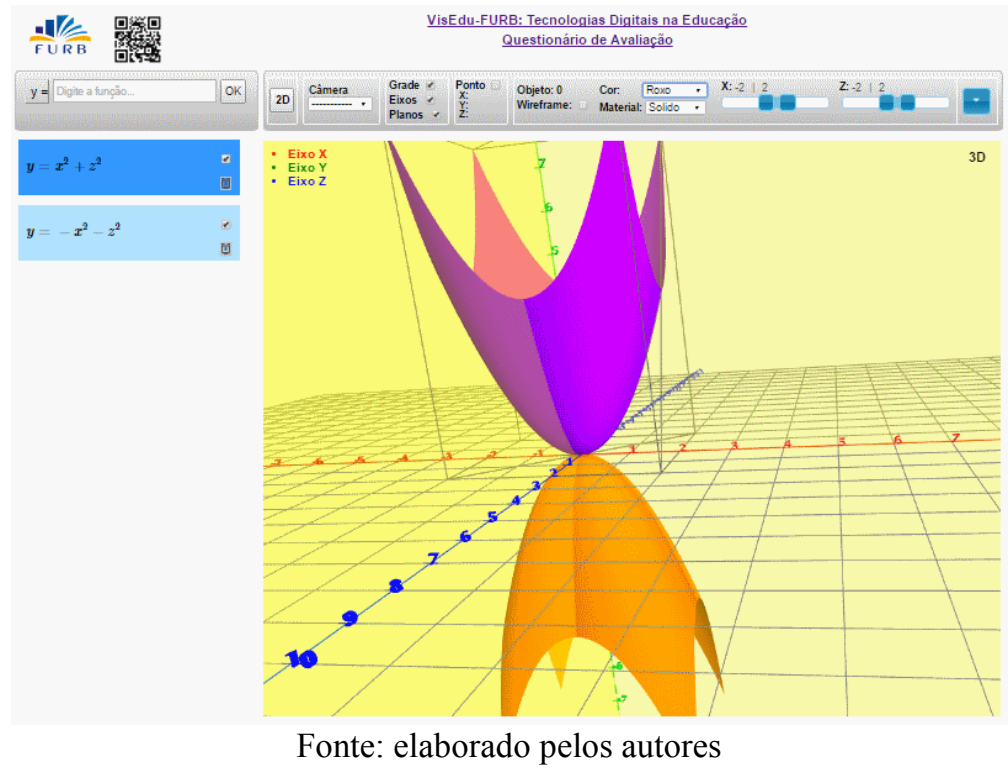

Figura 2. VISEDU-MAT - Espaço R3

\section{Resultados}

O VISEDU-MAT está sendo aplicado na Escola de Ensino Médio Professora Elza H.T. Pacheco, em Blumenau (SC). Até o momento o ambiente foi usado em sete turmas, sendo quatro do Primeiro ano, duas do Segundo e uma do Terceiro, envolvendo mais de 200 alunos. Ainda está prevista a aplicação em mais seis turmas. A atividade é realizada em notebooks com tela sensível ao toque do Laboratório Interdisciplinar de Formação de Educadores (LIFE), da FURB.

$\mathrm{Na}$ atividade, os estudantes são organizados em grupos de dois ou três alunos. Dependendo do ano da turma, a professora de Matemática, do Núcleo de Estudos de Ensino de Matemática (NEEM), da FURB, executa uma sequência didática de acordo com os conteúdos que os estudantes estão trabalhando. A atividade é executada em duas aulas de 45 minutos e é acompanhada por pelo menos um técnico de informática e uma monitora de matemática. No fim é aplicado um questionário de avaliação. Como trabalham em grupo, alguns alunos apresentam suas respostas coletivamente.

Foram coletadas 76 respostas, sendo exatamente metade de cada gênero. A maior parte dos respondentes (77,6\%) tem entre 15 e 17 anos. 36,8\% utilizam o computador mais de três horas por dia e $89,5 \%$ usam o smartphone mais de três horas por dia, indicando o uso intenso de tecnologias digitais pelos estudantes. A Tabela 1 apresenta os resultados de cinco questões feitas aos estudantes sobre o ambiente. Destaca-se que todos consideraram o mesmo intuitivo e fácil de usar, e as avaliações positivas, considerando as duas primeiras categorias de cada escala, aproximam-se ou superam $90 \%$. 
V Congresso Brasileiro de Informática na Educação (CBIE 2016)

Anais dos Workshops do V Congresso Brasileiro de Informática na Educação (CBIE 2016)

Tabela 1. Resultados da aplicação do VISEDU-MAT

\begin{tabular}{|c|c|c|}
\hline \multicolumn{2}{|c|}{ Qual é a sua avaliação da aplicação } & $\begin{array}{l}\text { O aplicativo auxiliou na aprendizagem sobre o conteúdo estudado } \\
\text { na sala de aula? }\end{array}$ \\
\hline Muito Bom & $65,8 \%$ & $60,5 \%$ \\
\hline Bom & $28,9 \%$ & Mais ou menos \\
\hline Regular & $5,3 \%$ & Pouco \\
\hline Insatisfatório & $0,0 \%$ & Não ajudou \\
\hline \multicolumn{2}{|c|}{$\begin{array}{l}\text { Das atividades solicitadas, quantas } \\
\text { você conseguiu executar? }\end{array}$} & $\begin{array}{l}\text { Esta atividade lhe auxiliou a relembrar os conteúdos matemáticos } \\
\text { explorados e a compreender melhor os conceitos envolvidos? }\end{array}$ \\
\hline Todas & $81,6 \%$ & $59,2 \%$ \\
\hline A maior parte & $14,5 \%$ & Mais ou menos \\
\hline Metade & $3,9 \%$ & Pouco \\
\hline Menos da metade & $0,0 \%$ & Não ajudou \\
\hline Nenhuma & $0,0 \%$ & De modo geral, você achou o ambiente intuitivo e fácil de usar? \\
\hline 1 & & $100 \%$ \\
\hline & & Não \\
\hline
\end{tabular}

Fonte: elaborado pelos autores

\section{Conclusões}

Muitas vezes, nas aulas de matemática, os alunos têm dificuldade em entender alguns conceitos que requerem a análise gráfica, pois os mesmos não são devidamente explorados devido à falta de tempo. O VISEDU-MAT auxilia neste processo, pois apresenta os gráficos de forma simples e rápida, possibilitando trabalhar aliando álgebra e geometria. Além disso, no R3 a visualização é facilitada pela possibilidade de rotacionar os gráficos, visualizando-os e analisando-os sob diversos ângulos.

Com base nos resultados de sua aplicação, o VISEDU-MAT mostrou ser um ambiente intuitivo e de fácil utilização. Ele possibilita a plotagem de várias funções ao mesmo tempo, diferenciando-as por cores. Além disso, trata-se de um ambiente gratuito e disponível na web. Assim, o VISEDU-MAT apresenta-se com grande potencial para auxiliar os professores em suas práticas de ensino e os estudantes na compreensão dos conceitos de funções matemáticas nos espaços R2 e R3.

\section{Referências}

Krauss, J. R. (2013). Visedu-mat: visualizador de material educacional, módulo de matemática. 63 f. Trabalho de Conclusão de Curso (Graduação em Ciência da Computação) - Universidade Regional de Blumenau, Blumenau.

Machado, O. B. (2014). Visedu-mat 2.0: visualizador de material educacional - módulo de matemática. $82 \mathrm{f}$. Trabalho de Conclusão de Curso (Graduação em Ciência da Computação) - Universidade Regional de Blumenau, Blumenau.

Montibeller, J. P. (2014). Visedu-cg: aplicação didática para visualizar material educacional, módulo de computação gráfica. $106 \mathrm{f}$. Trabalho de Conclusão de Curso (Graduação em Ciência da Computação) - Universidade Regional de Blumenau, Blumenau.

Nunes, S. A. (2014). Visedu-cg 3.0: aplicação didática para visualizar material educacional - módulo de computação gráfica. $89 \mathrm{f}$. Trabalho de Conclusão de Curso (Graduação em Ciência da Computação) - Universidade Regional de Blumenau, Blumenau. 